\title{
Lyapunov Stability and Strong Passivity Analysis for Nonlinear Descriptor Systems
}

\author{
Chunyu Yang, Member, IEEE, Jing Sun, Fellow, IEEE, Qingling Zhang, and Xiaoping Ma
}

\begin{abstract}
In this paper, Lyapunov stability and strong passivity are defined for nonlinear descriptor systems. The new concepts facilitate the formulation of the relationship between the stability and passivity of nonlinear descriptor systems. A Lyapunov stability theorem which describes a sufficient condition for the systems to be globally asymptotically stable and of index one is derived. By the Lyapunov stability theorem, the connection between Lyapunov stability and strong passivity is established. Furthermore, strong passivity of feedback systems is discussed and two passivity theorems are given. Using the given passivity theorems, strongly absolute stability of Lur'e type descriptor systems is discussed. The obtained strongly absolute stability criterion is shown to be more general and less conservative than the existing methods. Finally, two examples are used to illustrate the advantages and effectiveness of the obtained methods.
\end{abstract}

Index Terms-Lyapunov stability, nonlinear descriptor systems, strong passivity.

\section{INTRODUCTION}

D ESCRIPTOR systems (also referred to as singular, differential-algebraic equation, or generalized state-space systems) have attracted much attention for their extensive applications in the areas of chemical engineering, circuits, economics, mechanical systems, etc. [1], [2]. Many fundamental system theories developed for standard state-space systems have been successively generalized to its counterparts for descriptor systems, for example, controllability and observability [3], LQ problem [4], $H_{\infty}$ control [5], etc. However, stability and passivity of nonlinear descriptor systems and the relationship between them are open problems.

Manuscript received February 16, 2012; revised June 06, 2012; accepted June 29, 2012. Date of publication September 24, 2012; date of current version March 23, 2013. This work was supported by the National Natural Science Foundation of China $(60904009,60974004,60904079,61020106003)$, the Fundamental Research Funds for the Central Universities (N110408001), the National Basic Research Program of China (2009CB320601), the Funds for Creative Research Groups of China (60821063), and the 111 project (B08015). The original and a shortened version of this work was presented at 49th IEEE Conference on Decision and ControlAtlanta, GA. This paper was recommended by Associate Editor X. Li.

C. Yang is with the School of Information and Electrical Engineering, China University of Mining and Technology, Xuzhou, 221116, China and also with the State Key Laboratory of Synthetical Automation for Process Industries, Northeastern University, Shenyang, 110819, China (e-mail: chunyuyang@yahoo.cn).

J. Sun is with the Department of Electrical Engineering and Computer Science, University of Michigan Ann Arbor, MI 48109 USA (e-mail: jingsun@umich.edu).

Q. Zhang is with the Institute of Systems Science, Northeastern University, Shenyang, 110819, China (e-mail: qlzhang@mail.neu.edu.cn).

$\mathrm{X} . \mathrm{Ma}$ is with the School of Information and Electrical Engineering, China University of Mining and Technology, Xuzhou, 221116, China (e-mail: xpma@cumt.edu.cn).

Digital Object Identifier 10.1109/TCSI.2012.2215396
Stability plays a central role for most of the analysis and synthesis problems of dynamic systems. Lyapunov direct method (LDM) has been the most popular and efficient approach for stability analysis. However, for descriptor sytems, because of the inherent mixed differential-algebraic nature, the selection of Lyapunov function candidate (LFC) and the calculation of the derivative of the LFC along the motions of the systems are more difficult than those for standard state-space systems, which results in a challenging task for researchers. For linear descriptor systems, the so-called generalized LFC and LDM have been proposed. The related stability criteria are usually expressed by matrix rank conditions and matrix inequalities [6]-[8]. However, LDM is premature for nonlinear descriptor systems.

For nonlinear descriptor systems, stability results based on LFCs, which are positive definite with respect to the full state $x$, are not easy to use [9]. The existing stability results for nonlinear descriptor systems can be roughly divided into two classes. One class of the presented stability conditions, which concern stability properties of partial state or functions of state $x$, i.e. $q(t, x)$, are derived by using LFCs relating to $q(t, x)$ rather than the full state $x$ (see, e.g., [10]-[14]). The other class of the existing stability results deal with stability properties of the full state $x$ and are derived by using LFCs relating to the dynamic state and the relationship between the static and dynamic states (see, e.g., [15]-[19]).

It is known that a descriptor system may contain impulse modes which are undesirable since they tend to destroy the systems [6]-[8]. Impulse modes have been widely studied in the analysis and synthesis problems of linear descriptor systems (see, e.g., [1], [5]). The notion of index one for nonlinear descriptor systems can be thought of as the generalization of the impulse-free property of linear descriptor systems. Taking into account the importance of stability and index one, some investigations have considered them simultaneously. In [16], a sufficient condition for nonlinear descriptor systems to be locally asymptotically stable and of index one was proposed. In [18], [19], strongly absolute stability of Lur'e type descriptor systems was defined to be globally stable and of index one and some criteria were proposed.

Passivity relates nicely to stability of systems [20]-[22]. On one hand, storage functions induced by passivity are usually related with system energy and thus provide natural candidates for Lyapunov functions, on the other hand, passivity is expected to be preserved under feedback interconnection, which provides a useful tool for stability analysis of feedback systems. Passivity and passive control problems for linear descriptor systems have been widely considered [23]-[26]. However, few works have dealt with passivity of nonlinear descriptor systems except for 
[28], [29], where some basic concepts and theorems on dissipativity were generalized to nonlinear descriptor systems.

In this paper, we will consider full state stability and passivity of nonlinear descriptor systems, propose corresponding criteria for them and establish connection between them. First, based on the characteristics of descriptor systems, the classical concept on Lyapunov stability is refined for descriptor systems. A Lyapunov stability theorem which describes a sufficient condition for the system to be globally asymptotically stable and of index one is derived. Then, under the framework of dissipativity, strong passivity is defined for descriptor systems. The connection between Lyapunov stability and strong passivity is established by the proposed Lyapunov stability theorem. Furthermore, strong passivity of feedback systems is discussed and two passivity theorems are given. Finally, strongly absolute stability of Lur'e type descriptor systems (LDS) is investigated by using the given passivity theorems. It is shown that the proposed results are more general and less conservative than the existing ones. Two examples are presented to illustrate the obtained results.

The notations used here are standard in most respects. We use $R$ to denote the set of real numbers. $R^{n}$ and $R^{n_{1} \times n_{2}}$ are the obvious extensions to vectors and matrices of the specified dimensions. Let $I$ or $I_{r}$ denote the identity matrix of appropriate dimension. For matrix $M, M^{T}$ stands for the transpose of $M$. $\operatorname{Re}(\cdot)$ and $\operatorname{Im}(\cdot)$ denote the real part and the imaginary part of a complex number, respectively. $\operatorname{det}(\cdot)$ denotes the determinant of a matrix. $\operatorname{deg}(\cdot)$ represents the degree of a polynomial. $\|\cdot\|$ denotes the Euclidean norm of a vector or matrix. For an arbitrary matrix $W$ and two symmetric matrices $P$ and $Q$, the symmetric term in a symmetric matrix is denoted by $*$, that is,

$$
\left[\begin{array}{ll}
P & W \\
* & Q
\end{array}\right]=\left[\begin{array}{cc}
P & W \\
W^{T} & Q
\end{array}\right]
$$

\section{Stability ANALYsis OF NONLINEAR DESCRIPTOR SySTEMS}

In this section, we will refine the classical concept on Lyapunov stability and propose a Lyapunov stability theorem for nonlinear descriptor systems.

\section{A. Definitions of Stability for Nonlinear Descriptor Systems}

Consider a linear time-invariant descriptor system

$$
E \dot{x}=A x,
$$

where $x \in R^{n}$ is the state variable, $A, E \in R^{n \times n}$ are constant matrices and $\operatorname{rank}(E)=r \leq n$.

We state here some basic definitions which will be used in the sequel and can be found in [1] and [7]. If $\operatorname{det}(s E-A) \neq 0$ for some complex number $s$, then the pair $(E, A)$ is said to be regular. Regularity of $(E, A)$ guarantees the existence and uniqueness of the solutions to system (1). A regular pair $(E, A)$ is called impulse-free if $\operatorname{deg} \operatorname{det}(s E-A)=\operatorname{rank}(E)$. If all roots of $\operatorname{det}(s E-A)=0$ lie in $\operatorname{Re}(s)<0,(E, A)$ is called stable. And the pair $(E, A)$ is called admissible if it is regular, impulse-free and stable. It is proved in [6] that $(E, A)$ is regular if and only if there exist two nonsingular matrices $M$ and $N$ such that $(E, A)$ can be transformed into the Weierstrass canonical form

$$
M E N=\left[\begin{array}{cc}
I_{r} & 0 \\
0 & J
\end{array}\right], M A N=\left[\begin{array}{cc}
A_{1} & 0 \\
0 & I
\end{array}\right]
$$

where $J \in R^{(n-r) \times(n-r)}$ is a nilpotent matrix, $A_{1} \in R^{r \times r}$. And system $(E, A)$ is impulse-free if and only if $J=0$.

Consider the nonlinear descriptor system

$$
E \dot{x}=f(x),
$$

where $f: R^{n} \rightarrow R^{n}$ is smooth enough and $f(0)=0$.

Definition 2.1: [30] System (3) is of index one if the constant coefficient system

$$
E \dot{w}=f_{x}(\hat{x}) w
$$

is regular and impulse-free for all $\hat{x}$ in a neighborhood of the equilibrium point $x=0$, where $f_{x}$ is the Jacobian matrix $\partial f / \partial x$.

Solutions to descriptor systems are very complex because of the existence of algebraic constraints. This paper considers continuous solutions of system (3), although distributional solutions are also important. The initial conditions for continuous solutions are required to be consistent.

Definition 2.2: [31] The initial condition $x(0)=x_{0}$ is consistent at $t=0$ if there exists a solution $x(t)$ to system (3), such that $x(0)=x_{0}=\lim _{t \rightarrow 0^{+}} x(t)$.

As illustrated by [16], [32], knowledge of $E x(0)$ is sufficient to completely determine the solution $x(t)$ of system (3) for $t \geq$ 0 . Thus we introduce the following assumption on the existence and uniqueness of solutions to system (3).

Assumption 2.1: For any $E x_{0}$ with $x_{0}$ being a consistent initial condition, system (3) has unique continuous solution over $[0,+\infty)$.

In the sense of Lyapunov stability: an equilibrium point is stable if all solutions starting at nearby points stay nearby; otherwise, it is unstable. It is asymptotically stable if all solutions starting at nearby points not only stay nearby, but also tend to the equilibrium point as time approaches to infinity [20]. One can see that Lyapunov stability describes certain continuous dependence of the solutions on initial conditions. Motivated by this idea, we define the following stability concepts for system (3).

Definition 2.3: The equilibrium point $x=0$ of system (3) is

i) stable if, for each $\varepsilon>0$, there is $\delta=\delta(\varepsilon)>0$ such that

$$
\|\operatorname{Ex}(0)\|<\delta \Rightarrow\|x(t)\|<\varepsilon, \forall t \geq 0,
$$

where $x(0)$ denotes arbitrary consistent initial condition.

ii) locally asymptotically stable if it is stable and $\delta$ can be chosen such that

$$
\|\operatorname{Ex}(0)\|<\delta \Rightarrow \lim _{t \rightarrow+\infty} x(t)=0,
$$

where $x(0)$ denotes arbitrary consistent initial condition. 
iii) globally asymptotically stable if it is stable and for any consistent initial condition $x(0)$, it holds that

$$
\lim _{t \rightarrow+\infty} x(t)=0 .
$$

Remark 2.1: When $E=I$, system (3) is reduced to a standard state-space system and Definition 2.3 coincides with the corresponding stability definitions for a standard state-space system [20].

Remark 2.2: For a time-invariant descriptor system (1), asymptotical stability defined by Definition 2.3 is equivalent to the widely used definition, that is, all roots of $\operatorname{det}(s E-A)=0$ satisfying $\operatorname{Re}(s)<0$. To show this, we take Laplace transformation for system (1). Then we have $X(s)=(s E-A)^{-1} E x(0)$. Consequently, Assumption 2.1 is equivalent to requiring the system to be regular. Thus without loss of generality, we can assume that system (1) is in the Weierstrass canonical form

$$
\begin{aligned}
\dot{x}_{1} & =A_{1} x_{1}, \\
J \dot{x}_{2} & =x_{2},
\end{aligned}
$$

where $J \in R^{(n-r) \times(n-r)}$ is a nilpotent matrix, $A_{1} \in R^{r \times r}$. The solution of system (6) is given by

$$
\begin{aligned}
& x_{1}(t)=e^{A_{1} t} x_{1}(0), \\
& x_{2}(t)=0 .
\end{aligned}
$$

Thus, the consistent initial condition is given by $x(0)=$ $\left[x_{1}^{T}(0) x_{2}^{T}(0)\right]^{T}=\left[x_{1}^{T}(0) 0^{T}\right]^{T}$. Therefore, according to Definition 2.3 , system (6) is asymptotically stable if and only if every eigenvalue of $A_{1}$ has negative real part.

Remark 2.3: In [16], [28], the classical Lyapunov stability of nonlinear descriptor systems was studied. Specifically, system (3) is stable if for each $\hat{\varepsilon}>0$, there is $\hat{\delta}=\hat{\delta}(\hat{\varepsilon})>0$ such that

$$
\|x(0)\|<\hat{\delta} \Rightarrow\|x(t)\|<\hat{\varepsilon}, \forall t \geq 0,
$$

where $x(0)$ denotes arbitrary consistent initial condition. It can be seen that (5) implies (8). The inverse is also true if system (3) is of index one. To show this, without loss of generality, we assume that system (3) is in the following form

$$
\begin{aligned}
\dot{x}_{1} & =f_{1}\left(x_{1}, x_{2}\right), \\
0 & =f_{2}\left(x_{1}, x_{2}\right) .
\end{aligned}
$$

When system (9) is of index one, there exists a unique solution $x_{2}=h\left(x_{1}\right)$ in some neighborhood of the equilibrium $x(0)=0$ satisfying $f_{2}\left(x_{1}, h\left(x_{1}\right)\right)=0$ with $h(0)=0$. Then, $x_{1} \rightarrow 0 \Rightarrow x_{2} \rightarrow 0$. Hence, for any $\delta>0$, there is $\hat{\delta}>0$, such that $\|E x(0)\|=\left\|x_{1}(0)\right\| \leq \delta \Rightarrow\|x(0)\| \leq \hat{\delta}$, which shows that (8) implies (5). Thus the stability concept defined by Definition 2.3 is equivalent to the classical Lyapunov stability [16], [28] if the system is of index one. But the new concept facilitates the formulation of the relationship between the stability and passivity of nonlinear descriptor systems, as will be shown in the next section.

\section{B. Lyapunov Stability Theorem for Nonlinear Descriptor Systems}

In [16], [28], a sufficient condition was proposed for system (3) to be of index one and locally asymptotically stable in the sense of classical Lyapunov stability. According to the discussion in Remark 2.3, the stability in the sense of Definition 2.3 is equivalent to the classical Lyapunov stability if system (3) is of index one. Hence, the result is also valid in the sense of Definition 2.3. The following lemma recalls the above mentioned result.

Lemma 2.1: [16] Let $D \subset R^{n}$ be a neighborhood of $x=0$. Suppose that there exists a $C^{3}$ function $V: D \rightarrow R^{+}$vanishing at $E x=0$ and positive elsewhere which satisfies the following properties

i) $(\partial / \partial x) V=\hat{V}^{T}(x) E$ for some $C^{1}$ function $\hat{V}: X \rightarrow$ $R^{n}$

ii) $\dot{V}=\hat{V}(x) f(x)<0, \forall x \subset D, x \neq 0$;

iii) $E^{T} \hat{V}_{x}=\hat{V}_{x}^{T} E \geq 0, \forall x \subset D$, where $\hat{V}_{x}$ denotes the Jacobian of $\hat{V}$.

Then the equilibrium $x=0$ of system (3) is locally asymptotically stable and the nonlinear descriptor system is of index one.

To establish Lyapunov stability Theorem for nonlinear descriptor systems, we recall the following well-known definition and lemma.

Definition 2.4: [20] A continuous function $\alpha:[0,+\infty) \rightarrow$ $[0,+\infty)$ is said to belong to class $K$ if it is strictly increasing and $\alpha(0)=0$. A class $K$ function $\alpha(r)$ is said to belong to class $K_{\infty}$ if $\alpha(r) \rightarrow+\infty$ as $r \rightarrow+\infty$.

Lemma 2.2: [20] Let $W: R^{n} \rightarrow R$ be a continuous positive definite function. Then, there exist class $K$ functions $\alpha_{1}$ and $\alpha_{2}$, such that

$$
\alpha_{1}(\|x\|) \leq W(x) \leq \alpha_{2}(\|x\|), \forall x \in R^{n} .
$$

Moreover, if $W(x)$ is radially unbounded, then $\alpha_{1}$ and $\alpha_{2}$ can be chosen to belong to class $K_{\infty}$.

Now we are ready to propose the Lyapunov stability theorem for nonlinear descriptor system (3).

Theorem 2.1: Suppose that there exists a function $V(z)$ : $R^{n} \rightarrow R^{+}$with $z=E x$, satisfying the following properties:

i) $V(0)=0$;

ii) $V$ is continuously differentiable in $R^{n}$ and twice continuously differentiable in a neighborhood of the origin;

iii) $V(z)>0, \forall z \neq 0$;

iv) $(\partial / \partial z) V=\hat{V}^{T}(x) E$ for some function $\hat{V}: R^{n} \rightarrow R^{n}$;

v) $\dot{V}=\hat{V}^{T}(x) f(x)<0, \forall x \neq 0$;

vi) $E^{T} \hat{V}_{x}=\hat{V}_{x}^{T} E \geq 0$, where $\hat{V}_{x}$ denotes the Jacobian of $\hat{V}$

vii) $\lim _{\|z\| \rightarrow+\infty} V(z) \rightarrow+\infty$.

Then system (3) is globally asymptotically stable and of index one.

Proof: Using Lemma 2.1, conditions i)-vi) imply that the equilibrium $x=0$ is locally asymptotically stable and the nonlinear descriptor system is of index one. Consequently, according to Definition 2.3, the globally asymptotical stability is guaranteed if, for any small $\delta>0$, there exists $\bar{t}>0$, such 
that $\|E x(\bar{t})\|<\delta$. Thus, to show the globally asymptotical stability, it sufficient to prove $\lim _{t \rightarrow+\infty}\|E x(t)\|=0$.

For any given $z_{0}=E x_{0}$, let $c=V\left(z_{0}\right)$. Condition vii) implies that for any $c>0$, there exists $\gamma>0$ such that $V(z)>c$ whenever $\|z\|>\gamma$. Thus $V(z) \leq c \Rightarrow\|z\| \leq \gamma$.

Denote $\Omega=\{z \mid V(z) \leq c\}$. It follows from condition v) that

$$
V(z(t)) \leq V(z(0)) \leq c, \forall t \geq 0 .
$$

Then any trajectories starting in $\Omega$ at $t=0$ stay there for all $t \geq 0$.

Condition iii) and v) indicates that $V(z(t))$ is monotonically decreasing and bounded from below by zero, which shows that

$$
\lim _{t \rightarrow+\infty} V(z(t))=a \geq 0 .
$$

To show $a=0$, we use a contradiction argument. Suppose $a>$ 0 . By continuity of $V(z)$, there is $d$ with $0<d<\gamma$ such that $\|z\| \leq d \Rightarrow V(z) \leq 0.5 a$. Then $\lim _{t \rightarrow+\infty} V(z(t))=a>0$ implies that $\|z\|>d, \forall t \geq 0$.

By Lemma 2.2, it follows from conditions i)-iv) that there exist class $K_{\infty}$ functions $\alpha_{1}(\cdot), \alpha_{2}(\cdot)$, and class $K$ functions $\alpha_{3}(\cdot), \alpha_{4}(\cdot)$ such that

$$
\begin{aligned}
\alpha_{1}(\|z\|) & \leq V(z) \leq \alpha_{2}(\|z\|), \\
\dot{V} & \leq-\alpha_{3}(\|x\|) \leq-\alpha_{4}(\|z\|) \leq-\alpha_{4}(d) \triangleq-\beta .
\end{aligned}
$$

Since $\beta>0$, we have

$$
\begin{aligned}
V(z(t)) & =V(\operatorname{Ex}(t)) \\
& =V(\operatorname{Ex}(0))+\int_{0}^{t} \dot{V}(\operatorname{Ex}(\tau)) d \tau \\
& \leq V(\operatorname{Ex}(0))-\beta t .
\end{aligned}
$$

Since the right-hand side will eventually become negative, the inequality contradicts the assumption that $a>0$. Therefore, we have $\lim _{t \rightarrow+\infty} V(z(t))=0$ which shows that $\lim _{t \rightarrow+\infty}\|E x(t)\|=0$ and system (3) is globally asymptotically stable and of index one.

Remark 2.4: Theorem 2.1 generalizes and extends some of the existing stability results for descriptor systems.

- Consider the case that system (3) is a linear system in the form of (1). Define a generalized quadratic Lyapunov function $V(E x)=x^{T} E^{T} P E x=x^{T} E^{T}\left(P E+E_{0} Q\right) x$, where $P \in R^{n \times n}$ satisfying $P=P^{T}>0$, $Q \in R^{(n-r) \times n}, E_{0} \in R^{n \times(n-r)}$ is a matrix of full column rank such that $E^{T} E_{0}=0$ and $\operatorname{rank}(E)=r$. Then $V(E x)$ satisfies conditions i), ii), iii), iv), vi), and vii) of Theorem 2.1. And condition v) is equivalent to $A^{T}\left(P E+E_{0} Q\right)+\left(P E+E_{0} Q\right)^{T} A<0$. Therefore, Theorem 2.1 reduces to the Lyaponov stability theorem given by [8] if system (3) is a linear time-invariant descriptor system.

- Compared with the stability results for nonlinear descriptor systems in [16], [28], Theorem 2.1 introduces condition vii). Such a condition is necessary to guarantee the global stability of nonlinear descriptor systems.

\section{PASSivity ANAlysis of NONLINEAR DESCRIPTOR SYSTEMS}

In this section, strong passivity is defined for descriptor systems by specializing the storage function and supply rate of dissipativity [28], [29]. The connection between stability and strong passivity is established. Furthermore, strong passivity of feedback systems is discussed and two passivity theorems are given.

\section{A. Definitions of Passivity for Nonlinear Descriptor Systems}

Consider the nonlinear descriptor system

$$
\begin{aligned}
E \dot{x} & =f(x, u), \\
y & =h(x, u),
\end{aligned}
$$

where $f: R^{n \times m} \rightarrow R^{n}$ is smooth enough, $h: R^{n \times m} \rightarrow R^{m}$ is continuous and $f(0,0)=0, h(0,0)=0$.

Definition 3.1: [28], [29] System (10) is said to be dissipative if there exists a storage function $V(E x) \geq 0$ and a scalar supply rate $\omega(u, y)$ such that the dissipation inequality

$$
V(\operatorname{Ex}(T))-V(\operatorname{Ex}(0)) \leq \int_{0}^{T} \omega(u(t), y(t)) d t
$$

holds along all possible trajectories of system (10) starting at $\operatorname{Ex}(0)$, for any $T \geq 0$.

If the storage function $V(E x)$ is differentiable, then inequality (11) is equivalent to

$$
\dot{V} \leq \omega(u(t), y(t)), \forall t \geq 0 .
$$

Passivity is dissipativity with the supply rate $\omega(u(t), y(t))=$ $u^{T} y$. Furthermore, if there exists a positive definite function $\phi(x)$, such that

$$
\dot{V} \leq u^{T}(t) y(t)-\phi(x), \forall t \geq 0,
$$

then system (10) is called strictly passive.

Throughout the paper, we will consider the passivity with additional properties.

Definition 3.2: System (10) is said to be strongly passive if it is strictly passive and the storage function $V(z)$, where $z=E x$, satisfies

i) $V$ is continuously differentiable in $R^{n}$ and twice continuously differentiable in a neighborhood of the origin;

ii) $V$ is positive definite and radially unbounded with respect to $z$. That is, $V(0)=0, V(z)>0, \forall z \neq$ $0, \lim _{\|z\| \rightarrow+\infty} V(z) \rightarrow+\infty$;

iii) $E^{T} \hat{V}_{x}=\hat{V}_{x}^{T} E \geq 0$, where $\hat{V}: R^{n} \rightarrow R^{n}$ such that $(\partial / \partial z) V=\hat{V}^{T}(x) E$ and $\hat{V}_{x}$ denotes the Jacobian of $\hat{V}$.

Remark 3.1: When $E=I$, Definition 3.2 reduces to the concept of strong passivity of standard state-space systems given by [33]. The assumptions i), ii), and iii) posed on the storage function facilitate to establish the connection between the strong passivity and stability of nonlinear descriptor systems. These assumptions are always satisfied for linear descriptor systems because linear passive systems have quadratic storage functions [26]. 


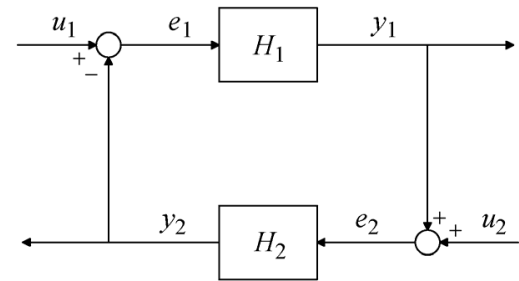

Fig. 1. Feedback connection.

Definition 3.3: [20] A function $h: R^{m} \rightarrow R^{m}$ is passive if $u^{T} h(u) \geq 0$.

\section{B. Passivity Theorem for Nonlinear Descriptor Systems}

Using Theorem 2.1, we have the following result which establishes the connection between stability and strong passivity of nonlinear descriptor systems.

Theorem 3.1: The origin of $E \dot{x}=f(x, 0)$ is globally asymptotically stable and of index one if system (10) is strongly passive.

Now we discuss strong passivity of feedback descriptor systems. Consider the feedback connection of Fig. 1, where each of the feedback components $H_{1}$ and $H_{2}$ is either a descriptor system of the following form

$$
H_{i}:\left\{\begin{array}{l}
E_{i} \dot{x}_{i}=f_{i}\left(x_{i}, e_{i}\right) \\
y_{i}=h_{i}\left(x_{i}, e_{i}\right), i=1,2,
\end{array}\right.
$$

or a function represented by

$$
y_{i}=h_{i}\left(e_{i}\right), i=1,2 \text {. }
$$

The interconnection is assumed to be well-defined.

When both components $H_{1}$ and $H_{2}$ are nonlinear descriptor systems, the closed-loop system takes the form of (10) with

$$
\begin{aligned}
& E=\left[\begin{array}{cc}
E_{1} & 0 \\
0 & E_{2}
\end{array}\right], x=\left[\begin{array}{l}
x_{1} \\
x_{2}
\end{array}\right], \\
& u=\left[\begin{array}{l}
u_{1} \\
u_{2}
\end{array}\right], y=\left[\begin{array}{l}
y_{1} \\
y_{2}
\end{array}\right] .
\end{aligned}
$$

In this case, the interconnection is well-defined if equation

$$
\begin{aligned}
& e_{1}=u_{1}-h_{2}\left(x_{2}, e_{2}\right) \\
& e_{2}=u_{2}+h_{1}\left(x_{1}, e_{1}\right)
\end{aligned}
$$

has a unique solution $\left(e_{1}, e_{2}\right)$ for every $\left(x_{1}, x_{2}, u_{1}, u_{2}\right)$.

When one component, say $H_{1}$ is a nonlinear descriptor system, while the other one is a function, the closed-loop system takes the form of (10) with

$$
x=x_{1}, u=\left[\begin{array}{l}
u_{1} \\
u_{2}
\end{array}\right], y=\left[\begin{array}{l}
y_{1} \\
y_{2}
\end{array}\right] .
$$

In this case, the interconnection is well-defined if equation

$$
\begin{aligned}
& e_{1}=u_{1}-h_{2}\left(e_{2}\right) \\
& e_{2}=u_{2}+h_{1}\left(x_{1}, e_{1}\right)
\end{aligned}
$$

has a unique solution $\left(e_{1}, e_{2}\right)$ for every $\left(x_{1}, u_{1}, u_{2}\right)$.

Theorem 3.2: The feedback connection of two strongly passive descriptor systems is strongly passive.

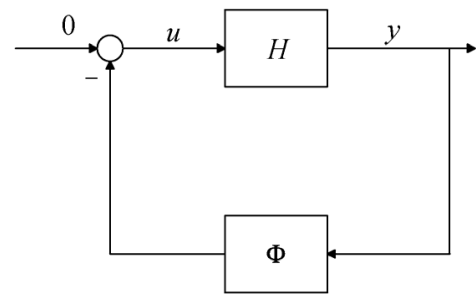

Fig. 2. Lur'e type descriptor systems.

Proof: Suppose that $H_{1}$ and $H_{2}$ are strongly passive. Then there exist storage functions $V_{1}\left(E_{1} x_{1}\right)$ and $V_{2}\left(E_{2} x_{2}\right)$ and positive definite functions $\phi_{1}\left(x_{1}\right)$ and $\phi_{2}\left(x_{2}\right)$ satisfying conditions i)-iii) of Definition 3.2 and $\dot{V}_{i} \leq e_{i}^{T} y_{i}-\phi_{i}\left(x_{i}\right)$.

From the feedback connection of Fig. 1, we have

$$
\begin{aligned}
e_{1}^{T} y_{1}+e_{2}^{T} y_{2} & =\left(u_{1}-y_{2}\right)^{T} y_{1}+\left(u_{2}+y_{1}\right)^{T} y_{2} \\
& =u_{1}^{T} y_{1}+u_{2}^{T} y_{2} .
\end{aligned}
$$

Let $V(E x)=V_{1}\left(E_{1} x_{1}\right)+V_{2}\left(E_{2} x_{2}\right)$ and $\phi(x)=\phi_{1}\left(x_{1}\right)+$ $\phi_{2}\left(x_{2}\right)$, we can show that $V(E x)$ satisfies conditions i)-iii) of Definition 3.2 and $\phi(x)$ is positive definite. Furthermore,

$$
\begin{aligned}
\dot{V} & =\dot{V}_{1}+\dot{V}_{2} \\
& \leq e_{1}^{T} y_{1}+e_{2}^{T} y_{2}-\phi_{1}\left(x_{1}\right)-\phi_{2}\left(x_{2}\right) \\
& =u^{T} y-\phi(x) .
\end{aligned}
$$

Hence, the feedback connection is strongly passive.

Similarly, we can have the following result.

Theorem 3.3: The feedback connection of a strongly passive descriptor system and a passive function is strongly passive.

Remark 3.2: Theorem 3.1 establishes the connection between Lyapunov stability and strong passivity of nonlinear descriptor systems. Theorems 3.2 and 3.3 generalize the classical passive theorems [20] to nonlinear descriptor systems. These results are useful for stability analysis of nonlinear feedback descriptor systems.

\section{Strongly Absolute Stability of LuR'e Type DESCRIPTOR SYSTEMS}

In the previous sections, Lyapunov stability theorem and passivity theorem are proposed for nonlinear descriptor systems. The results (Theorems 3.1 and 3.3) will be used in this section to further investigate strongly absolute stability of Lur'e type descriptor systems (LDS) that admit the block diagram representation of Fig. 2, which is the feedback interconnection of the descriptor system $H$ with a static nonlinearity $\Phi$.

The descriptor system $H$ is described by

$$
H:\left\{\begin{array}{l}
E \dot{x}=f(x, u), \\
y=h(x, u),
\end{array}\right.
$$

where $f(0,0)=0, h(0,0)=0$. The nonlinearity $\Phi$ satisfies certain sector condition.

The following definition summaries the sector terminology.

Definition 4.1: A function $\Phi: R^{m} \rightarrow R^{m}$ is said to belong to the sector

i) $[0,+\infty)$, if $y^{T} \Phi(y) \geq 0$;

ii) $\left[K_{1},+\infty\right)$, if $y^{T}\left(\Phi(y)-K_{1} y\right) \geq 0$, where $K_{1}$ is a symmetric positive definite matrix; 


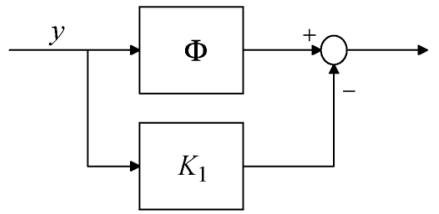

Fig. 3. Loop transform for the block $\Phi$ when $\Phi \in\left[K_{1},+\infty\right)$.

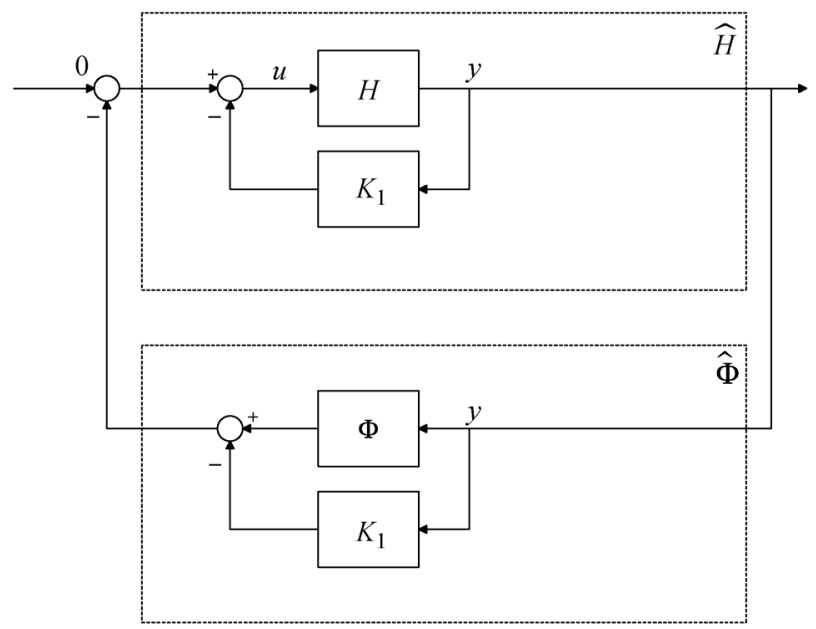

Fig. 4. Loop transformed Lur'e system when $\Phi \in\left[K_{1},+\infty\right)$.

iii) $\left[K_{1}, K_{2}\right]$ with $K=K_{2}-K_{1}=K^{T}>0$, if $(\Phi(y)-$ $\left.K_{1} y\right)^{T}\left(\Phi(y)-K_{2} y\right) \leq 0$, where $K_{1}$ and $K_{2}$ are symmetric matrices.

Definition 4.2: Consider the LDS, where $\Phi$ satisfies a sector condition in Definition 4.1. The system is said to be strongly absolutely stable if the equilibrium point is globally asymptotically stable and of index one for any nonlinearity in the given sector.

Remark 4.1: Definition 4.2 is a generalization of the classical absolute stability for standard state-space systems [20] as well as the strongly absolute stability for standard LDS where $H$ is a linear time-invariant descriptor system [18], [19].

We will propose a stability criterion for general LDS and then specialize the result to standard LDS.

\section{A. Strongly Absolute Stability Criterion for General LDS}

Theorem 4.1: Assume that $H$ is in the form of (16). Then, the LDS is strongly absolutely stable with respect to sector

i) $[0,+\infty)$, if system $H$ is strongly passive.

ii) $\left[K_{1},+\infty\right)$, if system $\hat{H}$ is strongly passive, where $\hat{H}$ is shown in Fig. 4.

iii) $\left[K_{1}, K_{2}\right]$, if system $\widetilde{H}$ is strongly passive, where $\widetilde{H}$ is shown in Fig. 6.

Proof: Assume that system $H$ is strongly passive. By Definition 4.1 , every $\Phi$ belonging to sector $[0,+\infty)$ is passive. Then, using Theorem 3.3, the feedback connection of system (16) and nonlinearity $\Phi \in[0,+\infty)$ is strongly passive. By Theorem 3.1 and Definition 4.2, the LDS is strongly absolutely stable with respect to sector $[0,+\infty)$. This completes the proof for $\mathrm{i})$.

As stated in [20], a function in sector $\left[K_{1},+\infty\right)$ can be transformed into a function in sector $[0,+\infty)$ by the loop transform described in Fig. 3. The transformed system described in Fig. 4 is equivalent to the original LDS. Then we can prove ii) by the same way as we prove i).

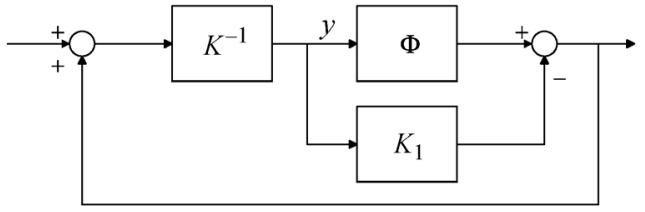

Fig. 5. Loop transform for the block $\Phi$ when $\Phi \in\left[K_{1}, K_{2}\right]$ with $K=K_{2}-$ $K_{1}$.

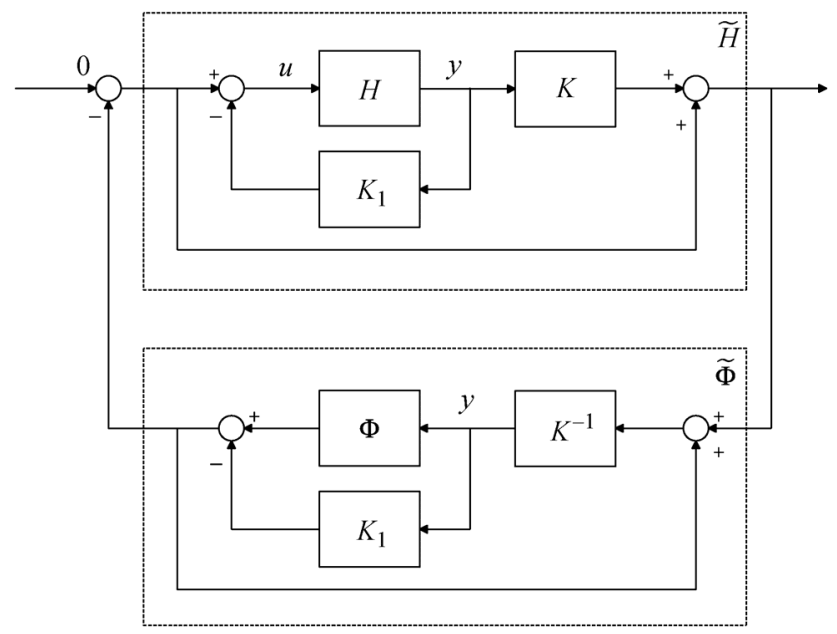

Fig. 6. Loop transformed Lur'e system when $\Phi \in\left[K_{1}, K_{2}\right]$ with $K=K_{2}-$ $K_{1}$.

A function in sector $\left[K_{1}, K_{2}\right]$ can be transformed into a function in sector $[0,+\infty)$ by the loop transform described in Fig. 5 . The transformed system described in Fig. 6 is equivalent to the original LDS. Then we obtain iii) by the same way as we prove i).

Remark 4.2: In [18], [19], strongly absolute stability problem for LDS was considered. However, the proposed results requires $H$ to be a linear time-invariant descriptor system and nonlinearity $\Phi$ to belong to $[0, K]$. Thus Theorem 4.1 is more general than the existing results.

\section{B. Strongly Absolute Stability Criterion for Standard LDS}

Consider the standard LDS, where $H$ is a linear time-invariant descriptor system,

$$
H:\left\{\begin{array}{l}
E \dot{x}=A x+B u, \\
y=C x+D u .
\end{array}\right.
$$

From Theorem 4.1, strongly absolute stability of LDS is guaranteed by the strong passivity of system $H$. The following result present a sufficient condition for (17) to be strongly passive.

Lemma 4.1: System (17) is strongly passive if there exist matrices $P \in R^{n \times n}, W \in R^{n \times m}$ satisfying the following LMI conditions

$$
\begin{aligned}
E^{T} P=P^{T} E & \geq 0, \\
E^{T} W & =0, \\
{\left[\begin{array}{cc}
A^{T} P+P^{T} A & A^{T} W+P^{T} B-C^{T} \\
* & W^{T} B+B^{T} W-D-D^{T}
\end{array}\right] } & <0 .
\end{aligned}
$$

Proof: Assume that there exist matrices $P \in R^{n \times n}, W \in$ $R^{n \times m}$ satisfying (18)-(20). 
Let $V(x)=0.5 x^{T} E^{T} P x$, where $P$ satisfies (18) and (20). It holds that $\operatorname{rank}\left(E^{T} P\right)=\operatorname{rank}(E)$ because matrix $P$ is nonsingular by (20). From [8], matrix $P$ satisfying $E^{T} P=P^{T} E \geq$ 0 and $\operatorname{rank}\left(E^{T} P\right)=\operatorname{rank}(E)$ can be parameterized by $P=$ $X E+E_{0} Q$, where $X \in R^{n \times n}$ satisfying $X=X^{T}>0$, $Q \in R^{(n-r) \times n}, E_{0} \in R^{n \times(n-r)}$ is a matrix of full column rank such that $E^{T} E_{0}=0$ and $\operatorname{rank}(E)=r$. As a result, $V(x)=0.5 x^{T} E^{T} P x=0.5 x^{T} E^{T} X E x$. Then $V(x)$ is a function of $E x$ and can be expressed as $V(E x)$. It is easy to show that $V(E x)$ satisfies conditions i)-iii) of Definition 3.2.

From (19), it follows that

$$
0=\dot{x}^{T} E^{T} W u=(A x+B u)^{T} W u .
$$

Differentiating $V(E x)$ along the trajectories of the system, we have

$$
\begin{aligned}
\dot{V}-u^{T} y= & 0.5\left[\dot{x}^{T} E^{T} P x+x^{T} P^{T} E \dot{x}\right]-u^{T}(C x+D u) \\
= & 0.5\left[(A x+B u)^{T} P x+x^{T} P^{T}(A x+B u)\right] \\
& -u^{T}(C x+D u) .
\end{aligned}
$$

Taking into account (21), we obtain

$$
\begin{aligned}
\dot{V}-u^{T} y= & 0.5\left[(A x+B u)^{T} P x+x^{T} P^{T}(A x+B u)\right] \\
& -u^{T}(C x+D u)+(A x+B u)^{T} W u \\
= & 0.5\left[\begin{array}{l}
x \\
u
\end{array}\right]^{T} \Theta\left[\begin{array}{l}
x \\
u
\end{array}\right]
\end{aligned}
$$

where

$$
\Theta=\left[\begin{array}{cc}
A^{T} P+P^{T} A & A^{T} W+P^{T} B-C^{T} \\
* & W^{T} B+B^{T} W-D-D^{T}
\end{array}\right] .
$$

From (20) and (23), there exists $\varepsilon>0$, such that

$$
\dot{V}-u^{T} y \leq-\varepsilon x^{T} x .
$$

Hence, system (17) is strongly passive.

Remark 4.3: If we set $W=0$ in (19) and (20), Lemma 4.1 reduces to Corollary 2 of [37], which provides a sufficient condition for admissibility and passivity of linear descriptor systems. Thus Lemma 4.1 is less conservative than the result of [37].

The LMI conditions in Lemma 4.1 coincide with those in Corollary 9 of [23], which provides a condition for admissibility as well as for extended strict positive realness. As stated by [23], non-strict LMIs may lead to numerical singularity problems. A method has been proposed by [23] to convert the non-strict LMI (18) into a strict one. In this paper, we will deal with the non-strict LMI (18) and (19), simultaneously, and show that the non-strict LMIs (18)-(20) can be converted into a single strict LMI.

Let orthogonal matrix $U=\left[\begin{array}{ll}U_{1} & U_{2}\end{array}\right]$ and $V=\left[V_{1} V_{2}\right]$ be such that

$$
E=U\left[\begin{array}{cc}
\Sigma_{r} & 0 \\
0 & 0
\end{array}\right] V^{T},
$$

where $\Sigma_{r} \in R^{r \times r}$ is positive definite and diagonal.

From (24), it can be seen that $E V_{2}=0$ and $U_{2}^{T} E=0$.

Then by (19), we have $V E^{T} U^{T} U^{-T} W=0$, which gives that $\left[\begin{array}{cc}\Sigma_{r} & 0 \\ 0 & 0\end{array}\right]\left[\begin{array}{c}\widehat{W} \\ W\end{array}\right]=0$ with $U^{-T} W=\left[\begin{array}{c}\widehat{W} \\ W\end{array}\right]$, where $\widehat{W} \in$ $R^{r \times m}$ and $\widetilde{W} \in R^{(n-r) \times m}$. As a result, $\widehat{W}=0$. Therefore, matrix variable $W$ satisfying (19) can be parameterized as

$$
W=U^{T}\left[\frac{0}{\widetilde{W}}\right]
$$

Furthermore, by [19], we have $Y_{1}=Y_{2}$ where $Y_{1}=\{P \in$ $\left.R^{n \times n} \mid E^{T} P=P^{T} E\right\}$ and $Y_{2}=\left\{P=X E+U_{2} S \mid X \in\right.$ $\left.R^{n \times n}, X=X^{T}, S \in R^{(n-r) \times n}\right\}$.

Thus, without any additional conservatism, the non-strict LMIs (18)-(20) in Lemma 4.1 can be converted to the following strict LMI,

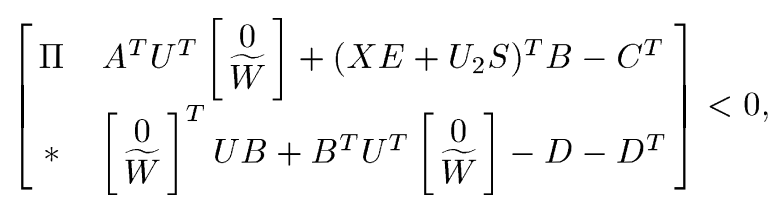

where $\Pi=A^{T}\left(X E+U_{2} S\right)+\left(X E+U_{2} S\right)^{T} A$.

Then by Lemma 4.1, we have the following result.

Lemma 4.2: System (17) is strongly passive if there exist a positive definite matrix $X \in R^{n \times n}$, and matrices $S \in R^{(n-r) \times n}, \widetilde{W} \in R^{(n-r) \times m}$ satisfying the strict LMI (25).

When $H$ is in the form of (17), the transformed system $\widehat{H}$ in Fig. 4 is given by

$$
\widehat{H}:\left\{\begin{array}{l}
E \dot{x}=\widehat{A} x+\widehat{B} u, \\
y=\widehat{C} x+\widehat{D} u,
\end{array}\right.
$$

where $\widehat{A}=A-B K_{1} C+B K_{1} D\left(I+K_{1} D\right)^{-1} K_{1} C, \widehat{B}=$ $B-B K_{1} D\left(I+K_{1} D\right)^{-1}, \widehat{C}=C, \widehat{D}=D$.

The transformed system $\widetilde{H}$ in Fig. 6 is given by

$$
\widetilde{H}:\left\{\begin{array}{l}
E \dot{x}=\widetilde{A} x+\widetilde{B} u, \\
y=\widetilde{C} x+\widetilde{D} u,
\end{array}\right.
$$

where $\widetilde{A}=A-B K_{1} C+B K_{1} D\left(I+K_{1} D\right)^{-1} K_{1} C, \widetilde{B}=$ $B-B K_{1} D\left(I+K_{1} D\right)^{-1}, \widetilde{C}=K C-K D\left(I+K_{1} D\right)^{-1} K_{1} C$, $\widetilde{D}=I+K D\left(I+K_{1} D\right)^{-1}$

Note that the existence of $\left(I+K_{1} D\right)^{-1}$ is necessary for the interconnection in Fig. 2 being well-defined.

Using Theorem 4.1 and Lemma 4.1, we have the following result.

Theorem 4.2: Assume $H$ is in the form of (17). Then, the LDS is strongly absolutely stable with respect to sector

i) $[0,+\infty)$, if there exist a positive definite matrix $X \in$ $R^{n \times n}$, and matrices $S \in R^{(n-r) \times n}, \widetilde{W} \in R^{(n-r) \times m}$ satisfying the strict LMI (25). 
ii) $\left[K_{1},+\infty\right)$, if there exist a positive definite matrix $X \in$ $R^{n \times n}$, and matrices $S \in R^{(n-r) \times n}, \widetilde{W} \in R^{(n-r) \times m}$ satisfying

$$
\left[\begin{array}{ll}
\widehat{\Pi} & \widehat{A}^{T} U^{T}\left[\frac{0}{\widetilde{W}}\right]+\left(X E+U_{2} S\right)^{T} \widehat{B}-\widehat{C}^{T} \\
* & \left.\left[\frac{0}{W}\right]^{T} U \widehat{B}+\widehat{B}^{T} U^{T}\left[\frac{0}{W}\right]-\widehat{D}-\widehat{D}^{T}\right]<0,
\end{array}\right.
$$

where $\widehat{\Pi}=\widehat{A}^{T}\left(X E+U_{2} S\right)+\left(X E+U_{2} S\right)^{T} \widehat{A}$.

iii) $\left[K_{1}, K_{2}\right]$, if there exist a positive definite matrix $X \in$ $R^{n \times n}$, and matrices $S \in R^{(n-r) \times n}, \widetilde{W} \in R^{(n-r) \times m}$ satisfying

$$
\left[\begin{array}{ll}
\widetilde{\Pi} & \widetilde{A}^{T} U^{T}\left[\frac{0}{\widetilde{W}}\right]+\left(X E+U_{2} S\right)^{T} \widetilde{B}-\widetilde{C}^{T} \\
* & \left.\left[\frac{0}{\widetilde{W}}\right]^{T} U \widetilde{B}+\widetilde{B}^{T} U^{T}\left[\frac{0}{\widetilde{W}}\right]-\widetilde{D}-\widetilde{D}^{T}\right]<0,
\end{array}\right.
$$

where $\widetilde{\Pi}=\widetilde{A}^{T}\left(X E+U_{2} S\right)+\left(X E+U_{2} S\right)^{T} \widetilde{A}$.

Remark 4.4: Theorem 4.2 is more general and less conservative than the existing methods [18], [35]. Specifically,

- The proposed Theorem 4.2 can deal with unbounded sector, but the methods in [18], [35] can not;

- When $\widetilde{W}=0$, iii) reduces to the stability conditions given by [18];

- When $\widetilde{W}=0$ and $D=0$, iii) reduces to the stability conditions given by [35].

Remark 4.5: Time-delays are often involved in practical control systems, which may induce instability, oscillations or bad performance for the closed-loop systems. Lur'e systems with time-delays have been widely considered (see [34] and the references therein). However, there are few investigations on LDS with time-delays. The exception is [36], where an absolute stability criterion was proposed by appropriate Lyapunov-Krasovskii functional construction. The proposed passivity framework in this paper provides a new idea for absolute stability analysis of LDS and is expected to be generalized to deal with absolute stability of LDS with time-delays, as will be considered in our future work.

\section{EXAMPLES}

In this section, examples are given to illustrate the proposed methods and show their advantages over the existing results.

\section{A. Example 1}

This example is borrowed from [18] to illustrate the advantages of the proposed methods over the existing results [18], [35]. The system matrices are as follows:

$$
\begin{aligned}
E & =\left[\begin{array}{llll}
2 & 0 & 0 & 0 \\
0 & 1 & 2 & 0 \\
1 & 0 & 1 & 0 \\
0 & 0 & 0 & 0
\end{array}\right], A=\left[\begin{array}{cccc}
-10 & 0 & 4 & 0 \\
0 & -10 & 2 & 0 \\
0 & 0 & -4 & 1 \\
0 & 2 & 0 & 1
\end{array}\right], \\
C & =\left[\begin{array}{llll}
0 & 1 & 1 & 0 \\
1 & 1 & 4 & 0
\end{array}\right], B^{T}=\left[\begin{array}{cccc}
1 & 1 & 0 & 0 \\
0 & 1 & 0 & -1
\end{array}\right], \\
D & =\left[\begin{array}{cc}
0 & 0 \\
0 & 0.5
\end{array}\right] .
\end{aligned}
$$

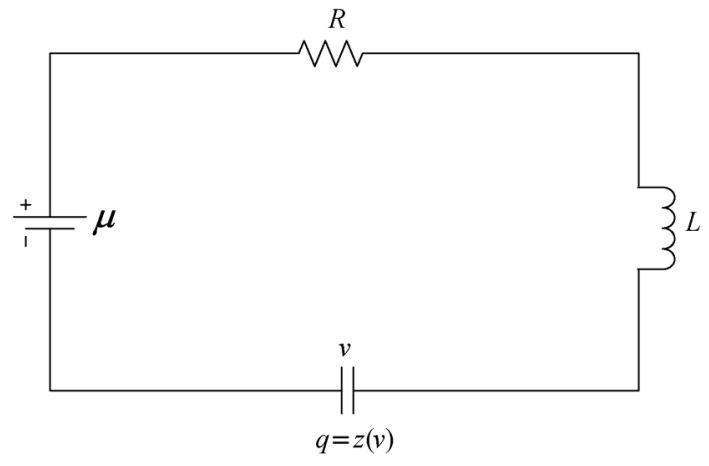

Fig. 7. A nonlinear circuit.

The method of [35] can not be used to analyze this example because the system matrix $D \neq 0$. As shown by [18], this system is strongly absolutely stable with respect to sector $[0, K]$ with $K=\left[\begin{array}{ll}2 & 1 \\ 1 & 1\end{array}\right]$. However, when $K=\left[\begin{array}{cc}10 & 1 \\ 1 & 1\end{array}\right]$, we find that the sufficient conditions presented in [18] are not feasible. We now consider this case by using Theorem 4.2.

By computation, $U, V$ and $\Sigma_{r}$ can be obtained by the singular value decomposition of $E$ as

$$
\begin{aligned}
U & =\left[\begin{array}{cccc}
-0.4318 & 0.7917 & -0.4321 & 0 \\
-0.7331 & -0.5872 & -0.3432 & 0 \\
-0.5255 & 0.1686 & 0.8339 & 0 \\
0 & 0 & 0 & 1.0000
\end{array}\right], \\
V & =\left[\begin{array}{cccc}
-0.5477 & 0.8328 & -0.0810 & 0 \\
-0.2890 & -0.2791 & -0.9157 & 0 \\
-0.7852 & -0.4781 & 0.3935 & 0 \\
0 & 0 & 0 & 1.0000
\end{array}\right], \\
\Sigma_{r}= & {\left[\begin{array}{cccc}
2.5365 & 0 & 0 & 0 \\
0 & 2.1038 & 0 & 0 \\
0 & 0 & 0.3748 & 0 \\
0 & 0 & 0 & 0
\end{array}\right] . }
\end{aligned}
$$

Solving LMI (29) gives

$$
\begin{aligned}
X & =\left[\begin{array}{cccc}
7.4210 & 3.6685 & -11.3342 & -0.0000 \\
3.6685 & 10.2067 & -12.4895 & 0.0000 \\
-11.3342 & -12.4895 & 33.6842 & -0.0000 \\
-0.0000 & 0.0000 & -0.0000 & 68.2247
\end{array}\right], \\
S & =\left[\begin{array}{llll}
-7.8081 & 32.3350 & -2.8988 & -15.4165
\end{array}\right], \\
\widetilde{W} & =\left[\begin{array}{cc}
0 & 0 \\
0 & 0 \\
0 & 0 \\
-0.5000 & 32.6123
\end{array}\right] .
\end{aligned}
$$

Then, by Theorem 4.2, the system is strongly absolutely stable with respect to sector $[0, K]$ with $K=\left[\begin{array}{cc}10 & 1 \\ 1 & 1\end{array}\right]$.

Therefore, as shown in Remark 4.4, Theorem 4.2 is more general and less conservative than the existing methods [18], [35].

\section{B. Example 2}

This example will demonstrate how the proposed method is applied to analyze the circuit displayed in Fig. 7, where a dc source with voltage $\mu$ is connected in series to a linear resistor, a linear inductor and a nonlinear capacitor with $q-v$ characteristic $q=z(v)$ satisfying sector constraint $\underline{m} \leq z(v) / v \leq \bar{m}$. Similar nonlinear capacitors have been considered in [38]. 
This circuit may be easily shown to admit the charge-flux description

$$
\begin{aligned}
\dot{q} & =\frac{\phi}{L}, \\
\dot{\phi} & =-\phi R / L-v+\mu, \\
0 & =-q+z(v),
\end{aligned}
$$

where $\phi$ is the magnetic flux in the inductor.

To study the stability of system (30) with $\mu=0$ by the proposed method, we shall rewrite the system as an LDS with $H$ in the form of (17). Let $x^{T}=\left[\begin{array}{lll}x_{1} & x_{2} & x_{3}\end{array}\right]=[q \phi v]$. Then we have $H$ in the form of (17) with

$$
\begin{aligned}
& E=\left[\begin{array}{lll}
1 & 0 & 0 \\
0 & 1 & 0 \\
0 & 0 & 0
\end{array}\right], A=\left[\begin{array}{ccc}
0 & 1 / L & 0 \\
0 & -R / L & -1 \\
-1 & 0 & 0
\end{array}\right], \\
& B=\left[\begin{array}{c}
0 \\
0 \\
-1
\end{array}\right], C=\left[\begin{array}{lll}
0 & 0 & 1
\end{array}\right], D=0 .
\end{aligned}
$$

The nonlinearity $\Phi(y)=z(v)$ and the section condition is described by $[\underline{m}, \bar{m}]$.

Let $R=1, L=1, \underline{m}=0.1, \bar{m}=10$.

Solving LMI (29), we have

$$
\begin{aligned}
X & =\left[\begin{array}{lll}
3.2077 & 0.0968 & 0.0000 \\
0.0968 & 0.2980 & 0.0000 \\
0.0000 & 0.0000 & 7.3074
\end{array}\right] \times 10^{2}, \\
S & =\left[\begin{array}{lll}
3.3397 & 2.4079 & -0.5083
\end{array}\right] \times 10^{2}, \\
\widetilde{W} & =\left[\begin{array}{lll}
0 & 0 & 1.4158
\end{array}\right]^{T} \times 10^{3} .
\end{aligned}
$$

Then, by Theorem 4.2, the system is strongly absolutely stable with respect to sector $[0.1,10]$. However, we find that the methods in [18], [35] are not valid for this example.

The presented examples show that Theorem 4.2 can be applied to more general LDS and is less conservative than the methods in [18], [35].

\section{CONCLUSIONS}

In this paper, the stability and passivity of nonlinear descriptor systems have been investigated. The classical concept on Lyapunov stability was refined and strong passivity was defined for descriptor systems, which facilitates to link the stability to passivity of nonlinear descriptor systems. A Lyapunov stability theorem which describes a sufficient condition for the system to be globally asymptotically stable and of index one was proposed. Furthermore, the connection between Lyapunov stability and strong passivity was established and two passivity theorems were given. Based on these results, strongly absolute stability criteria for Lur'e type descriptor systems were proposed, which were shown to improve the existing methods. Finally, two examples were given to demonstrate the utility of the methods and the advantages of the results.

The proposed results did not take into account time-delays which are often involved in practical control systems. In the future, we will try to extend the developed Lyapunov stability theorem and passivity theorem to deal with nonlinear descriptor systems with time-delays. In addition, $L_{2}$ stability of nonlinear descriptor systems, which relates nicely to passivity, is also an interesting topic for the future.

\section{REFERENCES}

[1] S. L. Campbell, Singular Systems of Differential Equations. London, U.K.: Pitman, 1980.

[2] M. D. S. Aliyu and E. K. Boukas, " $H_{\infty}$ filtering for nonlinear singular systems," IEEE Trans. Circuits Syst. I, Reg. Papers, 2012, 10.1109/ TCSI.2012.2189038.

[3] D. Cobb, "Controllability, observability and duality in singular systems," IEEE Trans. Autom. Control, vol. AC-29, no. 12, pp. 1076-1082, 1984.

[4] D. J. Bender and A. J. Laub, "The linear-quadratic optimal regulator for descriptor systems," IEEE Trans. Autom. Control, vol. AC-32, no. 8, pp. 672-688, 1987.

[5] I. Masubuchi, Y. Kamitane, A. Ohara, and N. Suda, " $H_{\infty}$ control for descriptor systems: A matrix inequalities approach," Automatica, vol. 33, no. 4, pp. 669-673, 1997.

[6] F. L. Lewis, "A survey of linear singular systems," Circuits, Syst., Signal Process., vol. 5, no. 1, pp. 3-36, 1986.

[7] L. Dai, Singular Control Systems. Berlin, Germany: Springer, 1989.

[8] J. Y. Ishihara and M. H. Terra, "On the Lyapunov theorem for singular systems," IEEE Trans. Autom. Control, vol. 47, no. 11, pp. 1926-1930, 2002.

[9] C. Ebenbauer and F. Allgower, "Stability analysis of constrained control systems: An alternative approach," Syst. Control Lett., vol. 56, no. 1, pp. 93-98, 2007.

[10] V. Dolezal, "Some practical stability criteria for semistate equations," Circuits, Syst., Signal Process., vol. 6, no. 3, pp. 335-354, 1987.

[11] V. B. Bajic, "Equations of perturbed motions and stability of state and semi-state systems," Int. J. Control, vol. 47, no. 6, pp. 1849-1860, 1988.

[12] B. B. Vladimir, "Lyapunov function candidates for semi-state systems," Int. J. Control, vol. 46, no. 6, pp. 2171-2181, 1987.

[13] C. Y. Yang, Q. L. Zhang, and L. N. Zhou, "Practical stability of descriptor systems with time delays in terms of two measurements," $J$. Franklin Inst., vol. 343, no. 6, pp. 635-646, 2006.

[14] C. Y. Yang, Q. L. Zhang, Y. P. Lin, and L. N. Zhou, "Practical stability of closed-loop descriptor systems," Int. J. Syst. Sci., vol. 37, no. 14, pp. 1059-1067, 2006.

[15] H. Wu and K. Mizukami, "Lyapunov stability theory and robust control of uncertain descriptor systems," Int. J. Syst. Sci., vol. 26, no. 10, pp. 1981-1991, 1995.

[16] H. Wang, C. Yung, and F. Chang, " $H_{\infty}$ control for nonlinear descriptor systems," IEEE Trans. Autom. Control, vol. 47, no. 11, pp. 1919-1925, 2002.

[17] G. Lu and D. W. C. Ho, "Generalized quadratic stability for continuous-time singular systems with nonlinear perturbation," IEEE Trans. Autom. Control, vol. 51, no. 5, pp. 818-823, 2006.

[18] C. Y. Yang, Q. L. Zhang, and L. N. Zhou, "Strongly absolute stability problem of descriptor systems," Informatica, vol. 18, no. 2, pp. 305-320, 2007.

[19] C. Y. Yang, Q. L. Zhang, and L. N. Zhou, "Strongly absolute stability of Lur'e descriptor systems: Popov type criteria," Int. J. Robust Nonlinear Control, vol. 19, no. 7, pp. 786-806, 2009.

[20] H. K. Khalil, Nonlinear Systems, 3rd ed. Upper Saddle River, NJ: Prentice-Hall, 2002.

[21] E. Steur and H. Nijmeijer, "Synchronization in networks of diffusively time-delay coupled (semi-)passive systems," IEEE Trans. Circiuts Syst. I, Reg. Papers, vol. 58, no. 6, pp. 1358-1371, 2011.

[22] J. L. Wang, H. N. Wu, and L. Guo, "Passivity and stability snalysis of reaction-diffusion neural networks with dirichlet boundary conditions," IEEE Trans. Neural Netw., vol. 22, no. 12, pp. 2105-2116, 2011.

[23] I. Masubuchi, "Dissipativity inequalities for continuous-time descriptor systems with applications to synthesis of control gains," Syst. Control Lett., vol. 55, no. 2, pp. 158-164, 2006.

[24] F. Chen and W. Zhang, "Robust passive control for uncertain singular systems with state delay," in Proc. 2006 Amer. Control Conf., Minneapolis, MN, 2006, pp. 1535-1538.

[25] M. K. Camlibel and R. Frasca, "Extension of Kalman-YakubovichPopov lemma to descriptor systems," Syst. Control Lett., vol. 58, no. 12, pp. 795-803, 2009. 
[26] Q. Li, Q. L. Zhang, N. Yi, and Y. H. Yuan, "Robust passive control for uncertain time-delay singular systems," IEEE Trans. Circiuts Syst. I, Reg. Papers, vol. 56, no. 3, pp. 653-663, 2009.

[27] I. Masubuchi, "Output feedback controller synthesis for descriptor systems satisfying closed-loop dissipativity," Automatica, vol. 43, no. 2, pp. 339-345, 2007.

[28] H. S. Wang, C. F. Yung, and F. R. Chang, $H_{\infty}$ Control for Nonlinear Descriptor Systems. London, U.K.: Springer-Verlag, 2006.

[29] N. A. Kablar, "Dissipativity theory for singular systems. Part I: Continuous-time case," in Proc. 42nd IEEE Conf. Decision Control, Seville, Spain, 2005, pp. 5639-5644.

[30] K. E. Brenan, S. L. Campbell, and L. R. Petzold, Numerical Solution of Initial-Value Problems in Differential-Algebraic Equations. Philadelphia, PA: SIAM, 1996.

[31] R. W. Newcomb, "The semistate description of nonlinear time-variable circuits," IEEE Trans. Circiuts Syst., vol. CAS-28, no. 1, pp. 62-71, 1981.

[32] C. V. George, C. L. Bernard, and K. Thomas, "A generalized statespace for singular systems," IEEE Trans. Autom. Control, vol. AC-26, no. 4, pp. 811-831, 1981

[33] G. B. Stan and R. Sepulchre, "Analysis of interconnected oscillators by dissipativity theory," IEEE Trans. Autom. Control, vol. 52, no. 2, pp. 256-270, 2007.

[34] J. Lu, J. Cao, and D. W. C. Ho, "Adaptive stabilization and synchronization for chaotic Lur'e systems with time-varying delay," IEEE Trans. Circiuts Syst. I, Reg. Papers, vol. 55, no. 5, pp. 1347-1356, 2008.

[35] C. Y. Yang, Q. L. Zhang, Y. P. Lin, and L. N. Zhou, "Positive realness and absolute stability problem of descriptor systems," IEEE Trans. Circiuts Syst. I, Reg. Papers, vol. 54, no. 5, pp. 1142-1149, 2007.

[36] H. Wang, A. Xue, and R. Lu, "Absolute stability criteria for a class of nonlinear singular systems with time delay," Nonlinear Anal., vol. 70, no. 3, pp. 621-630, 2009.

[37] Y. Gao, G. Lu, and Z. Wang, "Passive control for continuous singular systems with non-linear perturbations," IET Control Theory Appl., vol. 4, no. 11, pp. 2554-2564, 2010.

[38] R. Riaza, "Double SIB points in differential-algebraic systems," IEEE Trans. Autom. Control, vol. 48, no. 9, pp. 1625-1629, 2003.

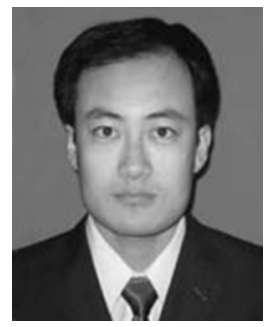

Chunyu Yang received his B.S. degree in applied mathematics and his Ph.D. degree in control theory and control engineering from Northeastern University, China, in 2002 and 2009, respectively.

$\mathrm{He}$ is currently an Associate Professor of China University of Mining and Technology, Xuzhou. His research interests include descriptor systems and robust control.

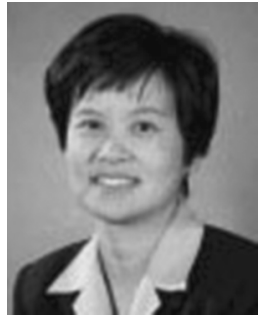

Jing Sun (F'04) received her Ph.D. degree from the University of Southern California, Los Angeles, in 1989, and her B.S. and M.S. degrees from the University of Science and Technology of China in 1982 and 1984, respectively.

From 1989-1993, she was an Assistant Professor in the Electrical and Computer Engineering Department, Wayne State University, Detroit, MI. She joined Ford Research Laboratory in 1993 where she worked in the Powertrain Control Systems Department. After spending almost 10 years in industry, she came back to academia and joined the faculty of the College of Engineering at the University of Michigan, Ann Arbor, in 2003, where she is now a Professor in the Department of Naval Architecture and Marine Engineering and Department of Electrical Engineering and Computer Science. Her research interests include system and control theory and its applications to marine and automotive propulsion systems. She holds 37 U.S. patents and has coauthored a textbook on robust adaptive control.

Dr. Sun is one of the three recipients of the 2003 IEEE Control System Technology Award.

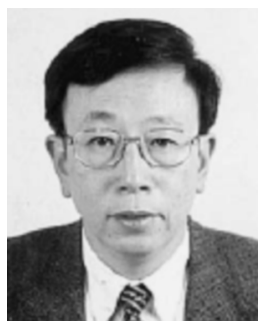

Qingling Zhang received his B.S. and M.S. degrees from the Mathematics Department and the Ph.D. degree from the Automatic Control Department of Northeastern University, Shenyang, China, in 1982, 1986 , and 1995 , respectively.

He finished his two year postdoctoral work in the Automatic Control Department of Northwestern Polytechnical University, Xian, China, in 1997. Since then, he has been a Professor at Northeastern University, Shenyang, China. He is also a member of the University Teaching Advisory Committee of National Ministry of Education. He has published six books and more than 230 papers about control theory and applications.

Dr. Zhang received 14 prizes from central and local governments for his research. He has also received the Golden Scholarship from Australia in 2000. During these periods, he visited Hong Kong University, Sydney University, Western Australia University and Niigata University, Pohan University of Science and Technology, Seoul University, Alberta University, Lakehead University, and Wisor University as a Research Associate, Research Fellow, Senior Research Fellow, and Visiting Professor, respectively.

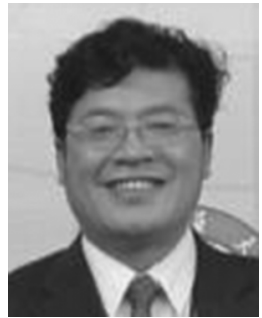

Xiaoping Ma received his B.S., M.S., and Ph.D. degrees from the School of Information and Electrical Engineering of China University of Mining and Technology, Xuzhou, in 1982, 1989, and 2001, respectively.

Now he is a Professor at China University of Mining and Technology, Xuzhou. His research interests include process control, networked control system, and fault detection. 